\title{
Effects of Bu Shen Yi sui capsule on NogoA/NgR and its signaling pathways RhoA/ROCK in mice with experimental autoimmune encephalomyelitis
}

Ling Fang ${ }^{1,3+}$, Yongqiang Wang ${ }^{1+}$, Qi Zheng ${ }^{1,4}$, Tao Yang ${ }^{2}$, Peiyuan Zhao ${ }^{1}$, Hui Zhao ${ }^{1}$, Qiuxia Zhang ${ }^{1}$, Yuanyuan Zhao ${ }^{5}$, Fang $\mathrm{Qi}^{1}$, Kangning $\mathrm{Li}^{2}$, Zhenzhen Chen ${ }^{1}$, Junling $\mathrm{Li}^{1}$, Nan Zhang ${ }^{1}$, Yongping Fan ${ }^{2}$ and Lei Wang ${ }^{1 *}$

\begin{abstract}
Background: Axon growth inhibitory factors NogoA/Nogo receptor (NgR) and its signaling pathways RhoA/ Rho kinase (ROCK) play a critical role in the repair of nerve damage in multiple sclerosis (MS). Bu Shen Yi Sui Capsule (BSYSC) is an effective Chinese formula utilized to treat MS in clinical setting and noted for its potent neuroprotective effects. In this study, we focus on the effects of BSYSC on promoting nerve repair and the underlying mechanisms in mice with experimental autoimmune encephalomyelitis (EAE), an animal model of MS.

Methods: The EAE mouse model was induced by injecting subcutaneously with myelin oligodendrocyte glycoprotein (MOG) $35-55$ supplemented with pertussis toxin. BSYSC was orally administrated at dose of 3. $0 \mathrm{~g} / \mathrm{kg}$ once a day for 40 days. The levels of protein gene product (PGP) 9.5, p-Tau, growth associated protein (GAP) -43, KI67 and Nestin in the brain or spinal cord on 20 and 40 day post-induction (dpi) were detected via immunofluorescence and Western blot analysis. Furthermore, NogoA/NgR and RhoA/ROCK signaling molecules were studied by GRT-PCR and Western blot analysis.

Results: Twenty or 40 days of treatment with BSYSC increased markedly PGP9.5 and GAP-43 levels, reduced p-Tau in the brain or spinal cord of mice with EAE. In addition, BSYSC elevated significantly the expression of KI67 and Nestin in the spinal cord 40 dpi. Further study showed that the activation of NogoA/NgR and RhoA/ROCK were suppressed by the presence of BSYSC.
\end{abstract}

Conclusions: BSYSC could attenuate axonal injury and promote repair of axonal damage in EAE mice in part through the down-regulation of NogoA/NgR and RhoA/ROCK signaling pathways.

Keywords: Experimental autoimmune encephalomyelitis, Multiple sclerosis, Bu Shen Yi sui capsule, PGP9.5, P-tau, Gap-43, Kl67, Nestin, NogoA/NgR, RhoA/ROCK

* Correspondence: tmwangl@ccmu.edu.cn

${ }^{\dagger}$ Equal contributors

${ }^{1}$ School of Traditional Chinese Medicine, Beijing Key Lab of TCM Collateral

Disease Theory Research, Capital Medical University, Beijing 100069, People's

Republic of China

Full list of author information is available at the end of the article 


\section{Background}

Multiple sclerosis (MS) is a central nervous system (CNS) autoimmune mediated inflammatory neurodegenerative disease [1], which is characterized by pathologic inflammation, demyelination, axonal degeneration and neuronal injury [2-4]. Recently, a large number of epidemiological studies confirm that annual global incidence and prevalence rate of MS increase progressively over the past years. MS predominantly occurs in young women, manifested with damages in the brain, spinal cord and the optic nerve, which eventually leads to sensory dysfunction, motor function disability and blindness in patients, even at early disease stages [5]. Experimental autoimmune encephalomyelitis (EAE) is very similar with human MS in pathological changes which is widely recognized as an animal model in MS research [6].

Growing evidence showed that demyelination and axonal damage is the major cause of neurological disability in MS patients, especially early occur axonal damage in the disease $[7,8]$. Therefore, the treatment of axonal repair is of great clinical significance in MS. With severe axonal injury in brain and spinal cord occurs in MS/EAE, the self repair function is activated to partially restore neurological function through a certain way in the early stage of axonal injury [9]. However, the natural recovery is far from enough to compensate for damaged axonal function. The research found that axon growth inhibitory factors play an important role in the failure of axonal regeneration. The inhibitors such as NogoA, myelin-associated glycoprotein (MAG) and oligodendrocyte myelin glycoprotein (OMgp) bind to a common Nogo-66 receptor (NgR) [10]. NgR signaling requires a p75 (NTR) or TROY in combination with an adaptor LINGO-1. Thus, each of the inhibitors interacts in $\mathrm{NgR}$ and its co-receptors to transduce the inhibitory signal into neurons [11], activating guanosine triphosphatase (GTPase), increasing the activity of the neuronal cytoskeleton regulatory factor RhoA, resulting in neuronal growth cone collapse and inhibiting the extension and regeneration of axons [12-15].

Since the precise pathogenesis of MS is unclear, there is no safe and effective therapeutics yet [16]. Traditional Chinese medicine (TCM) in treatment for MS gives promising results $[17,18]$. In addition to the previous studies, we found that BSYSC had a good effect in the treatment of MS $[19,20]$. The neuroprotective effect of BSYSC was found in EAE animal model [21-23]. However, the mechanism was not understood. In this study, the effects of BSYSC on axonal repair and regulation of axon growth inhibitory factors $\mathrm{NogoA} / \mathrm{NgR}$ and its signaling pathways RhoA/ROCK in EAE mice were explored. The results revealed a molecular mechanism, at least in partial, of BSYSC on axonal regeneration, and provided scientific evidence supporting its clinical application in treatment of MS.

\section{Methods}

\section{Animals}

The experimental protocol was approved by the Ethics Committee of Capital Medical University (No. 2011-X001). Specific pathogen-free (SPF) grade female C57BL/6 mice weighting 16-18 g, 6-to-8-week-old, were purchased from Beijing Vital River Laboratories, China [certification NO. SCXK (JING) 2006-0009]. The mice were kept in the Center of Laboratory Animals at Capital Medical University [certification NO. SYXK (JING) 2010-0020]. The mice were housed under a 12-h light/dark cycle in individual ventilated cages and maintained in a SPF grade environment.

\section{BSYSC preparation}

BSYSC consisted of ten crude herbs including Rehmanniae Radix, Rehmanniae radix Praeparata, Polygoni Multiflori Radix,Rhei Radix et Rhizoma, Leonuri Herba, Fritillariae Thunbergii Bulbus, Hirudo, Scorpio, Gastrodiae Rhizoma and Forsythiae Fructus at a ratio of 10:10:10:2:10:6:3:2:3:6. All of the herbal medicines in BSYSC were originally obtained from Beijing Ya Dong Biological Pharmacy Co., Ltd. (Beijing, China). Validation specimens were deposited at the Brain Disease Laboratory of the School of Traditional Chinese Medicine, Capital Medical University. The crude herbs were identified by associate Prof. Rong Luo in accordance with the Pharmacopoeia of People's Republic of China 2015 (1st set) [24]. The main components and chemical characteristic fingerprinting of BSYSC were identified by ultraperformance liquid chromatography-quadrupole time-offlight mass spectrometry (UPLC-QTOF-MS). The contents of acteoside and forsythiaside were detected, which were not less than $0.7 \mathrm{mg} / \mathrm{g}$ and $0.6 \mathrm{mg} / \mathrm{g}$, respectively, the same batch of BSYSC was used in the experiment. The detailed preparation procedure and quality control of BSYSC has been described in our recent report [23].

\section{Drugs and reagents}

Prednisone acetate (PA) was purchased from Zhejiang Xianju Pharmaceutical Co., Ltd. (Zhejiang, China). Myelin oligodendrocyte glycoprotein (MOG) $35-55$ (MEV GWYRSPFSRVVHLYRNGK, purity was >95\%) was synthesized by Beijing SciLight Biotechnology Co., Ltd. (Beijing, China). Mycobacterium tuberculosis H37Ra was purchased from Difco Co., Ltd. (Franklin Lakes, NJ, USA). Complete Freund' adjuvant (CFA) and pertussis toxin (PTX) were purchased from Sigma-Aldrich (St. Louis, MO, USA). Protein gene product (PGP) 9.5, Tau (phospho S262) and growth associated protein (GAP) -43, KI67 rabbit anti-mouse antibodies were purchased from Abcam Co., Ltd. (Cambridge, UK), protein Nestin mouse antimouse antibodies were purchased from Abcam Co., Ltd. (Cambridge, UK). NogoA rabbit anti- mouse antibody was purchased from Abcam Co., Ltd. (Cambridge, UK). NgR 
was purchased from Millipore Co., Ltd. (Massachusetts, USA). RhoA was purchased from Cell Signaling Technology Co., Ltd. (Boston, USA). ROCKII was from Abcam Co., Ltd. (Cambridge, UK). Alexa Fluor 488-labeled goat anti-rabbit, goat anti-mouse and 594-labeled goat anti-rabbit IgG $(\mathrm{H}+\mathrm{L})$ antibody were purchased from Jackson Co., Ltd. (Jackson, USA), GAPDH goat anti-rabbit IgG $(\mathrm{H}+\mathrm{L})$ antibody was purchased from Abcam Co., Ltd. (Cambridge, UK). The detailed contents are shown in Table 1. Western blot (WB) kits were purchased from Applygen Technologies Inc. (Beijing, China). Real-time quantitative reverse transcription-polymerase chain reaction (qRT-PCR) kits and reverse transcription kits were purchased from Tiangen Biotech Co., Ltd. (Beijing, China). PCR primers were synthesized by TaKaRa Biotechnology Co. Ltd. (Dalian, China).

\section{Induction and treatment of EAE}

Mice were randomly divided into four groups: normal control (NC, $n=20)$, EAE model (MO, $n=20)$, EAE + PAtreated (PA, $n=20)$ and EAE + BSYSC-treated (BSYSC, $n=20)$. The mice with EAE were immunized subcutaneously with $50 \mu \mathrm{g}$ of $\mathrm{MOG}_{35-55}$ emulsified in CFA containing $400 \mu \mathrm{g}$ MTB. PTX (500 ng) was followed by intraperitoneal injection on 0 and 2 day post-induction (dpi) [25]. The same volume of PBS was injected into a parallel group of mice as NC. For treatment of mice with medicines, mice in BSYSC group were given orally $3.0 \mathrm{~g} / \mathrm{kg}$ BSYSC once a day for 40 days. The dose of BSYSC was more effective against EAE in our previous study [22-24]. Mice in PA group were administered orally PA at a dose of $6 \mathrm{mg} / \mathrm{kg}$ after the EAE onset (the ninth day). Mice in NC and EAE groups were treated with equal amounts of distilled water.

\section{Sample collection}

The mice were sacrificed 20 dpi (acute stage, neurological function scores at a peak) and $40 \mathrm{dpi}$ (remission stage, no

Table 1 The antibodies used in the experiment

\begin{tabular}{llll}
\hline Name & Host & Dilution & Company \\
\hline PGP 9.5 & Rabbit & IF: 1:300 WB: 1:80000 (brain); 1:50000 & Abcam \\
(spinal cord) & & \\
p-Tau & Rabbit & IF: 1:50 WB: 1:20000 (brain); 1:10000 & Abcam \\
(spinal cord) & Abcam \\
GAP-43 & Rabbit & WB: 1:4000 & Abcam \\
KI67 & Rabbit & IF: 1: 100 & Abcam \\
Nestin & Mouse & IF: 1: 100 & Abcam \\
NogoA & Rabbit & WB: 1:20000 (brain); 1:2500 (spinal cord) & Abcalipore \\
NgR & Rabbit & WB: 1:2000 (brain); 1:5000 (spinal cord) & Millipol \\
RhoA & Rabbit & WB: 1:20000 (brain); 1:5000 (spinal cord) & Cell Signaling \\
ROCK & Rabbit & WB: 1:5000 & Abcam \\
GAPDH & Goat & WB: 1:2000 & Abcam \\
\hline
\end{tabular}

further increase in the signs of EAE). The brain and spinal cord of 5 mice was stored in $4 \%$ paraformaldehyde to be used for immunofluorescence (IF) analysis, and the brain and spinal cord of another 5 mice were immediately frozen for qRT-PCR and Western blot analyses.

\section{IF analysis}

The brain and spinal cord of mice were formalin-fixed, conventionally dehydrated, embedded in paraffin, and cut into serial sections. The sections were dewaxed, and boiled with citric acid at $95{ }^{\circ} \mathrm{C}$ for $20 \mathrm{~min}$. Then the sections were cooled to $30{ }^{\circ} \mathrm{C}$, and blocked with $10 \%$ goat serum at $37{ }^{\circ} \mathrm{C}$ for $60 \mathrm{~min}$. Subsequently the sections were incubated with primary detection antibody [rabbit anti-mouse PGP9.5 (1:300 dilution), rabbit anti-mouse p-Tau (1:50 dilution), rabbit anti-mouse KI67 (1:100 dilution), mouse anti-mouse Nestin (1:100 dilution)] at $4^{\circ}$ $\mathrm{C}$ for $48 \mathrm{~h}$. After $37{ }^{\circ} \mathrm{C}$ rewarming for $60 \mathrm{~min}$, the sections were incubated with the biotin-labeled secondary antibody [Alexa Fluor 488-labeled goat anti-rabbit IgG $(\mathrm{H}+\mathrm{L})$, revealing PGP9.5 (1:1000 dilution), p-Tau (1:400 dilution), goat anti-mouse IgG $(\mathrm{H}+\mathrm{L})$, revealing Nestin(1:400 dilution) and Alexa Fluor 594-labeled goat anti-rabbit IgG $(\mathrm{H}+\mathrm{L})$, revealing KI67 (1:400 dilution)] at $37{ }^{\circ} \mathrm{C}$ for $60 \mathrm{~min}$, then mounted with DAPIFluoromount-G, and stored at $4{ }^{\circ} \mathrm{C}$. Finally, the sections were observed under a Leica DM4000B fluorescence microscope (Leica, Solms, Germany). The image analysis was performed using Leica Qwin analysis software (Leica). Quantitative analysis was carried out with a NISElements BR 3.0 system. Five high-power fields $(\times 400)$ were selected in each sample and positive results were expressed as integrated optical density (IOD) values.

\section{QRT-PCR analysis}

NogoA, NgR, RhoA and ROCK mRNA expression in brain and spinal cord of mice were observed by qRT-PCR. In short, total RNA from approximately $30 \mathrm{mg}$ tissues was extracted according to the manufacturer's instructions. The cDNA was obtained from total RNA by the reverse transcription of $1 \mu \mathrm{g}$ of total RNA by the reverse transcription kit. The PCRs were performed with the following reaction protocol: $95{ }^{\circ} \mathrm{C}$ for $15 \mathrm{~min}$, followed by 40 cycles of denaturation at $95{ }^{\circ} \mathrm{C}$ for $30 \mathrm{~s}$, annealing at $54{ }^{\circ} \mathrm{C}$ for $10 \mathrm{~s}$ and extension at $72{ }^{\circ} \mathrm{C}$ for $10 \mathrm{~s}$ (Applied Biosystems 7300, Foster City, CA, USA). The relative quantification (RQ) was analyzed using the $2^{-\Delta \Delta \mathrm{Ct}}$ method. The sequences of primers were designed for RT-PCR using GenBank Accession sequences as follows: NogoA forward, 5' -CTTGG TCATGTGAACAGCACAATAA-3', reverse, 5' -CATTGA ACAAGGCACCAACGTAA-3'; NgR forward, 5'-TCCA GTCATGCCGAAATCTCAC-3', reverse, 5'-TGGTAGG GTCCACGACATGAAG-3'; RhoA forward, 5'-GGAGTG TTCAGCAAAGACCAAAG-3', reverse, 5' -CACAAGA 
TGAGGCACCCAGA-3'; ROCK forward, 5'-GGTATC GTCACAAGTAGCAGCATCA-3', reverse, 5'-TAAACC AGGGCATCCAATCCA-3'; $\beta$-actin forward, 5'-CATCCG TAAAGACCTCTATGCCAAC-3', reverse, 5' -ATGGAGC CACCGATCCACA-3'. $\beta$-actin mRNA served as an internal control. The amplified fragments were 124, 144, 111, 140 and 171 base pairs (bp), respectively.

\section{Western blotting analysis}

Protein expressions of PGP9.5, p-Tau, GAP-43, NogoA, NgR, RhoA and ROCK in brain and spinal cord of mice were detected by Western blot according to standard protocols. Briefly, $20 \mu \mathrm{g}$ proteins in each sample were processed by $5 \%$ and $10 \%$ SDS-PAGE and transferred onto a $0.45 \mu \mathrm{m}$ polyvinylidene fluoride membrane (Millipore, USA). The membranes were incubated with primarily anti-PGP9.5 antibody (brain 1:80,000, spinal cord 1:50,000 dilution), anti-pTau antibody (brain 1:20,000, spinal cord 1:10,000 dilution), anti-GAP-43 antibody (1:4000 dilution), anti-NogoA antibody (brain 1:20,000, spinal cord 1:2500 dilution), anti-NgR antibody (brain 1:2000, spinal cord 1:5000 dilution), antiRhoA antibody (brain 1:20,000, spinal cord 1:5000 dilution), anti-ROCK antibody (1:5000 dilution), or anti-GAPDH antibody (1:2000 dilution) as controls, followed by incubation with secondary goat anti-rabbit IgG $(1: 20,000)$ and electrochemiluminescence $(\mathrm{ECL})$ reagent for $1 \mathrm{~min}$. The membrane was exposed to Kodak film (Japan). Data were expressed by the integrated optical density (IOD) ratio using Image J (National Institutes of Health, USA).

\section{Statistical analysis}

The data were represented as means \pm standard error (SE). The data with normally distributed and equal variances were examined using one-way ANOVA with a post-hoc LSD test. Otherwise, the data were performed thyc $=10$ ? $>$ rank-sum test. The family-wise error rate was controlled by the statistical method of Bonferroni. The differences were considered significant at a value of $p<0.05$.

\section{Results}

Effects of BSYSC on the protein expression of PGP9.5 in EAE mice

IF analysis showed that PGP9.5 protein expression in the brain and spinal cord 20 and 40 dpi was reduced significantly in MO mice as compared with the NC mice $(p<0.01)$. The treatment with PA and BSYSC increased markedly PGP9.5 levels in the densitometry quantification as compared with the MO mice $(p<0.01$, Fig. 1a, b).

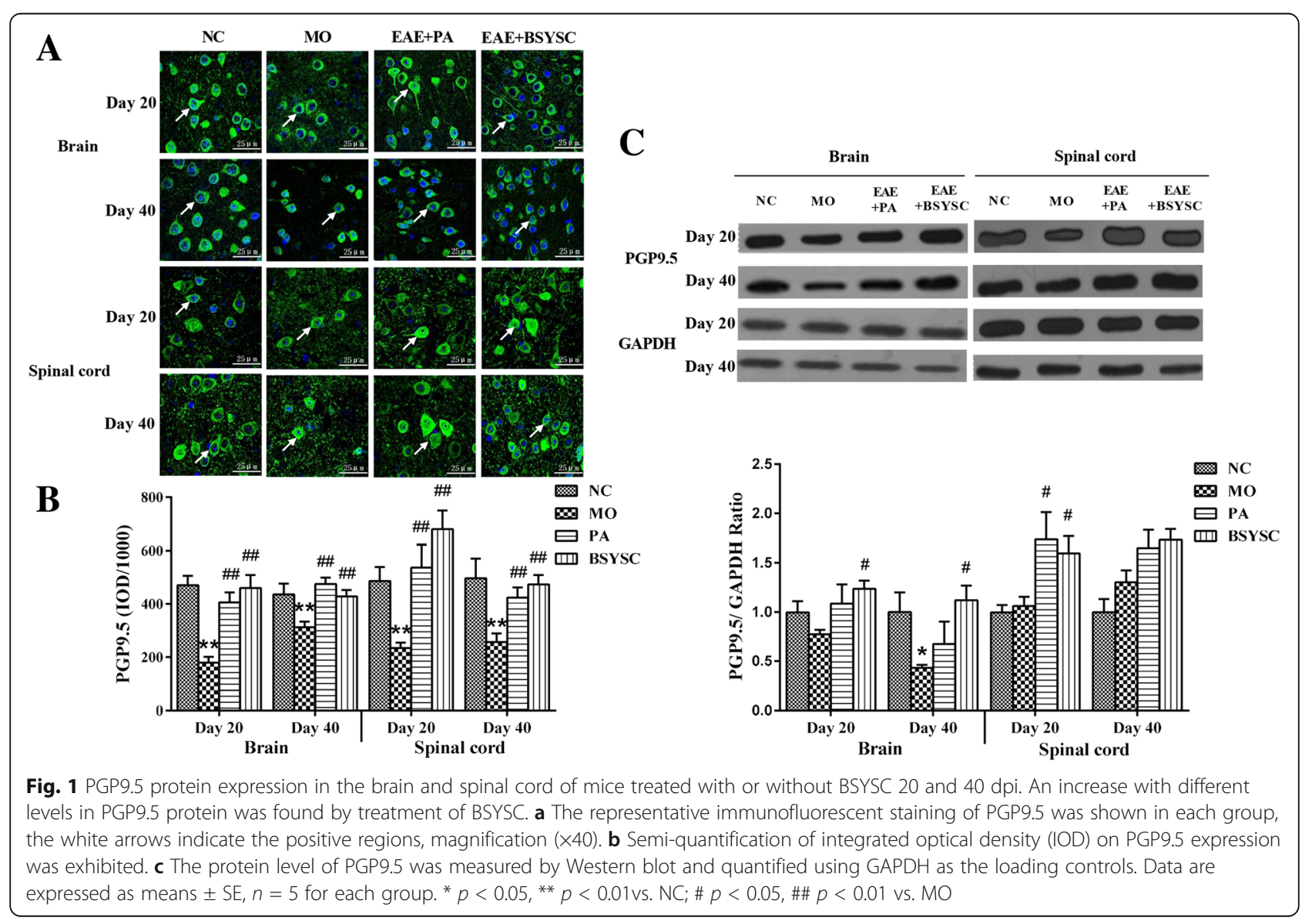


Western blot analysis demonstrated that PGP9.5 protein in the brain $40 \mathrm{dpi}$ was only reduced markedly in the $\mathrm{MO}$ mice as compared with the NC mice $(p<0.05)$. A significant increase in PGP9.5 protein of the brain 20 and $40 \mathrm{dpi}$ was observed in BSYSC mice as compared with the MO mice $(P<0.05)$. The increased PGP9.5 in the spinal cord 20 dpi was also shown in both PA and BSYSC mice as compared with the MO mice ( $p<0.05$, Fig. 1c).

\section{Effects of BSYSC on the protein expression of p-Tau in EAE mice}

In the IF analysis, a significant increase on $\mathrm{p}$-Tau protein expression in the brain and spinal cord 20 and $40 \mathrm{dpi}$ in MO mice as compared with NC mice $(p<0.01)$. However, p-Tau protein in the brain $20 \mathrm{dpi}$ was reduced significantly in BSYSC mice as compared with the MO mice $(p<0.01)$, and it also obviously reduced in the brain $40 \mathrm{dpi}$ in both PA and BSYSC mice $(p<0.01)$. While no significant difference in p-Tau protein of the spinal cord 20 and $40 \mathrm{dpi}$ in both PA and BSYSC mice (Fig. 2a, b). Western blot analyses showed that $\mathrm{p}$-Tau at the protein levels appeared a significant increase in the brain $40 \mathrm{dpi}$ and the spinal cord 20 dpi in MO mice as compared with NC mice $(p<0.05$ or $p<0.01)$. Whereas a significant reduction in $\mathrm{p}$-Tau protein of the brain 20 and 40 dpi was observed in BSYSC mice as compared with the MO mice $(p<0.05)$, and downregulation of p-Tau protein of spinal cord 20 dpi was also shown in both PA and BSYSC mice ( $p<0.01$, Fig. 2 c).

\section{Effects of BSYSC on the protein expression of GAP-43 in EAE mice}

No significant difference on GAP-43 protein expression was found between the $\mathrm{NC}$ and $\mathrm{MO}$ mice in western blot analysis. GAP- 43 protein in the brain $20 \mathrm{dpi}$ and the spinal cord $40 \mathrm{dpi}$ was increased in BSYSC mice as compared with the MO mice $(P<0.05)$. The increase of GAP-43 protein of the brain 20 and 40 dpi in BSYSC mice appeared to be larger than that of PA mice $(P<0.05$, Fig. 3).

\section{Effects of BSYSC on the protein expression of KI67 and Nestin in EAE mice}

The fluorescent imaging analysis showed that KI67 and Nestin protein expression in the spinal cord 20 and 40 dpi in the densitometry quantification was increased significantly in $\mathrm{MO}$ mice as compared with the $\mathrm{NC}$ mice $(P<0.001)$. Compared with the MO mice, KI67 and Nestin levels 20 dpi was reduced markedly by treatment

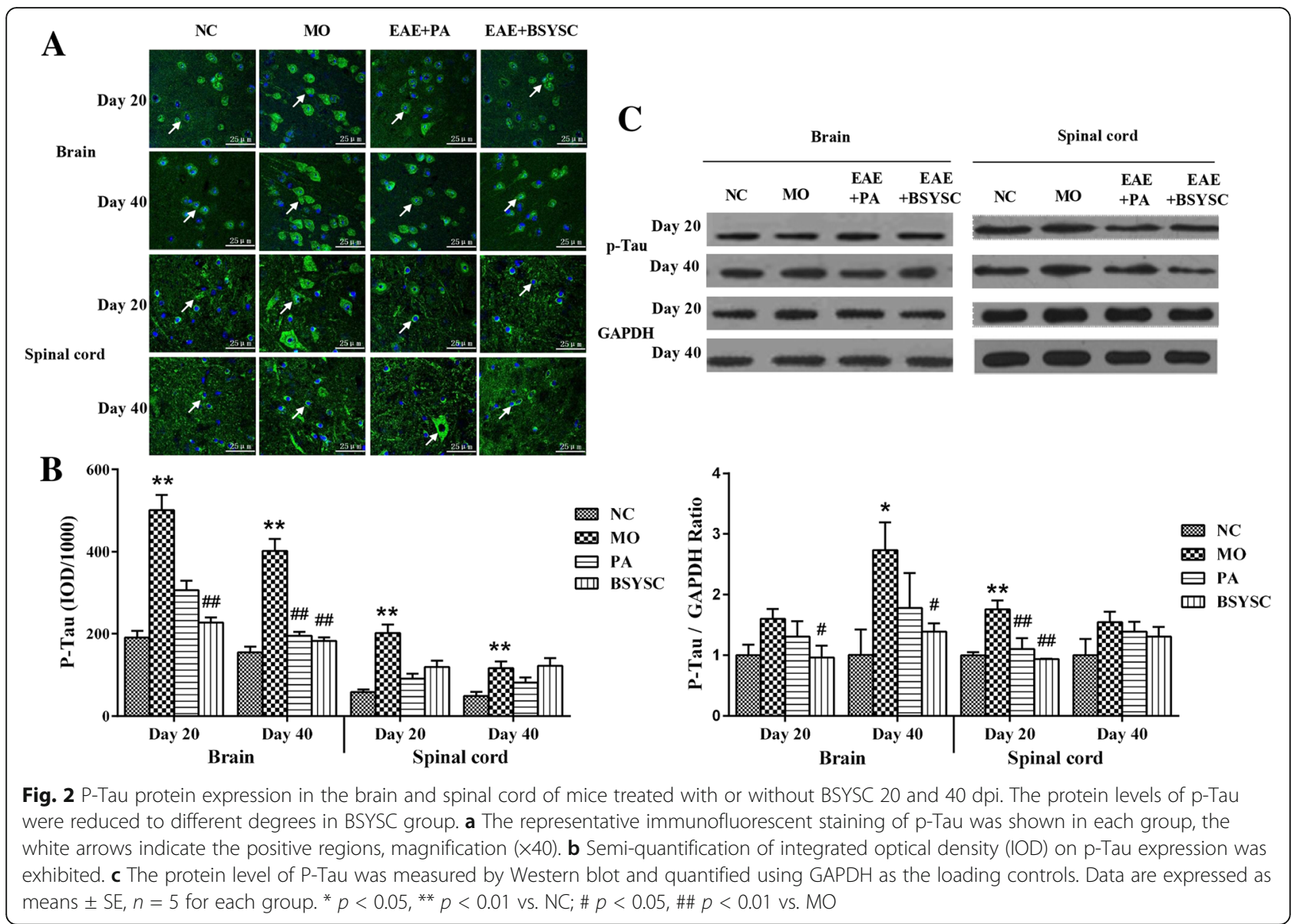



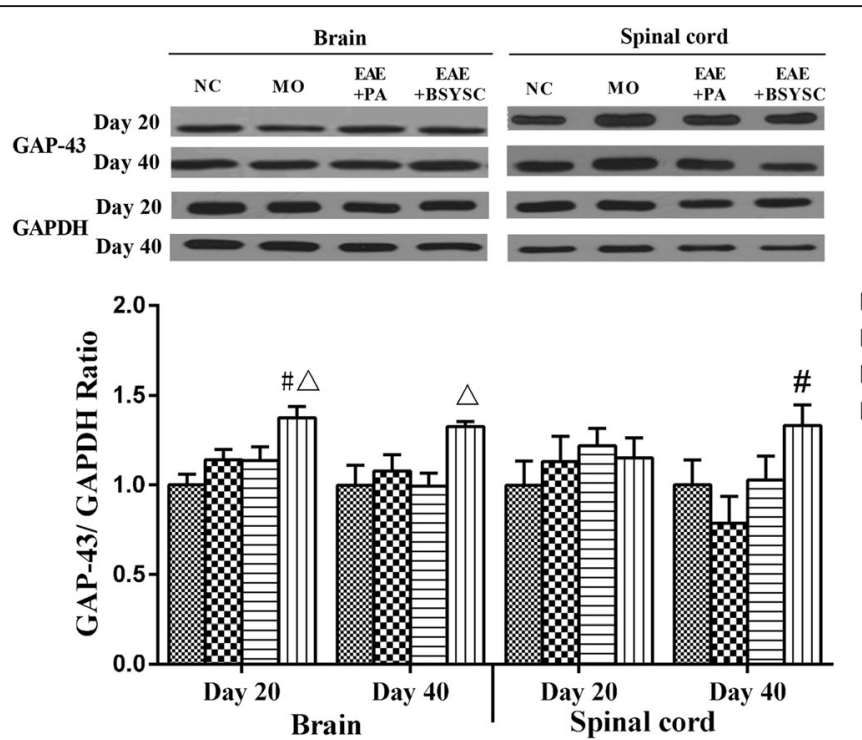

Fig. 3 GAP-43 protein expression in the brain and spinal cord of mice treated with or without BSYSC 20 and 40 dpi. A significant increase in GAP-43 protein was found in the brain 20 dpi and in the spinal cord 40 dpi in BSYSC group. The protein level of GAP-43 was measured by Western blot and quantified using GAPDH as the loading controls. Data are expressed as means $\pm \mathrm{SE}, n=5$ for each group. \# $p<0.05 \mathrm{vs}$. MO; $\Delta p<0.05$ vs. PA

with PA and BSYSC $(P<0.001$ or $P<0.01$ or $P<0.05)$, a significant increase in KI67 and Nestin $40 \mathrm{dpi}$ was observed in BSYSC or PA mice $(P<0.01$ or $P<0.001$, Fig. 4a, b and Fig. 5a, b).

\section{Effects of BSYSC on the mRNA and protein expressions of NogoA/NgR in EAE mice}

The qRT-PCR results showed that NogoA mRNA in brain 20 and 40 dpi significantly increased in the MO mice as compared with the NC mice $(P<0.05$ or $P<0.01)$, whereas the levels of NogoA mRNA were decreased significantly in the brain $40 \mathrm{dpi}$ and the spinal cord $20 \mathrm{dpi}$ in BSYSC mice as compared with the MO mice $(P<0.05)$, and it was also decreased in spinal cord 40 dpi in both PA and BSYSC mice $(P<0.05$, Fig. 6a). Compared with the NC mice, NgR mRNA was significantly increased in brain and spinal cord 20 and 40 dpi in the MO mice as compared with the NC mice $(P<0.05$ or $P<0.01)$. Decreased amounts of $\mathrm{NgR}$ mRNA were found in brain $40 \mathrm{dpi}$ in both PA and BSYSC mice as compared with the MO mice $(P<0.05$ or $P<0.01$ ), and NgR mRNA it was also decreased in the spinal cord 20 dpi in BSYSC mice $(P<0.01$, Fig. 6b).

Western blot analyses showed that NogoA protein was increased markedly, specifically in brain $40 \mathrm{dpi}$ in the MO mice as compared with the NC mice $(P<0.01)$. Decreased NogoA levels were observed in brain and spinal cord 20 and $40 \mathrm{dpi}$ in BSYSC mice $(P<0.05)$, and it also decreased in spinal cord $20 \mathrm{dpi}$ in PA mice $(P<0.05)$. In addition, BSYSC treatment seemed to perform better than PA as in the result of spinal cord 40 dpi $(P<0.05$, Fig. 6c). NgR was increased significantly at the protein level in spinal cord $40 \mathrm{dpi}$ as compared with the $\mathrm{NC}$ mice $(P<0.05)$. A significant reduction of $\mathrm{NgR}$ protein in brain and spinal cord 20 dpi was observed in both PA and BSYSC mice as compared with the MO mice $(P<0.05$ or $P<0.01)$, and NgR protein was also decreased in the brain and spinal cord $40 \mathrm{dpi}$ in BSYSC mice $(P<0.05$, Fig. 6d).

Effects of BSYSC on mRNA and the protein expressions of RhoA and ROCK in EAE mice

The qRT-PCR results showed that a significant increase of RhoA mRNA in the brain 20 and 40 dpi in the MO mice when compared with the NC mice $(P<0.01$ or $P<0.05)$. The obvious decreased RhoA mRNA in the brain and spinal cord 20 and 40 dpi was shown in both PA and BSYSC mice as compared with the MO mice $(P<0.05$, Fig. 1a). ROCK mRNA also appeared significant increase in brain and spinal cord 20 and 40 dpi in the MO mice as compared with the NC mice $(P<0.05)$. Decreased ROCK mRNA levels were observed in brain and spinal cord 20 and $40 \mathrm{dpi}$ in BSYSC mice $(P<0.05$ or $P<0.01)$, and it also decreased in brain $20 \mathrm{dpi}$ and spinal cord 40 dpi in PA mice $(P<0.01$, Fig. $7 b)$.

Western blot analyses showed that RhoA protein level significantly increased, specifically in brain $40 \mathrm{dpi}$ and in spinal cord 20 dpi in the MO mice as compared with the NC mice $(p<0.05$ or $p<0.01)$. Decreased RhoA were observed in brain and spinal cord 20 and 40 dpi in 


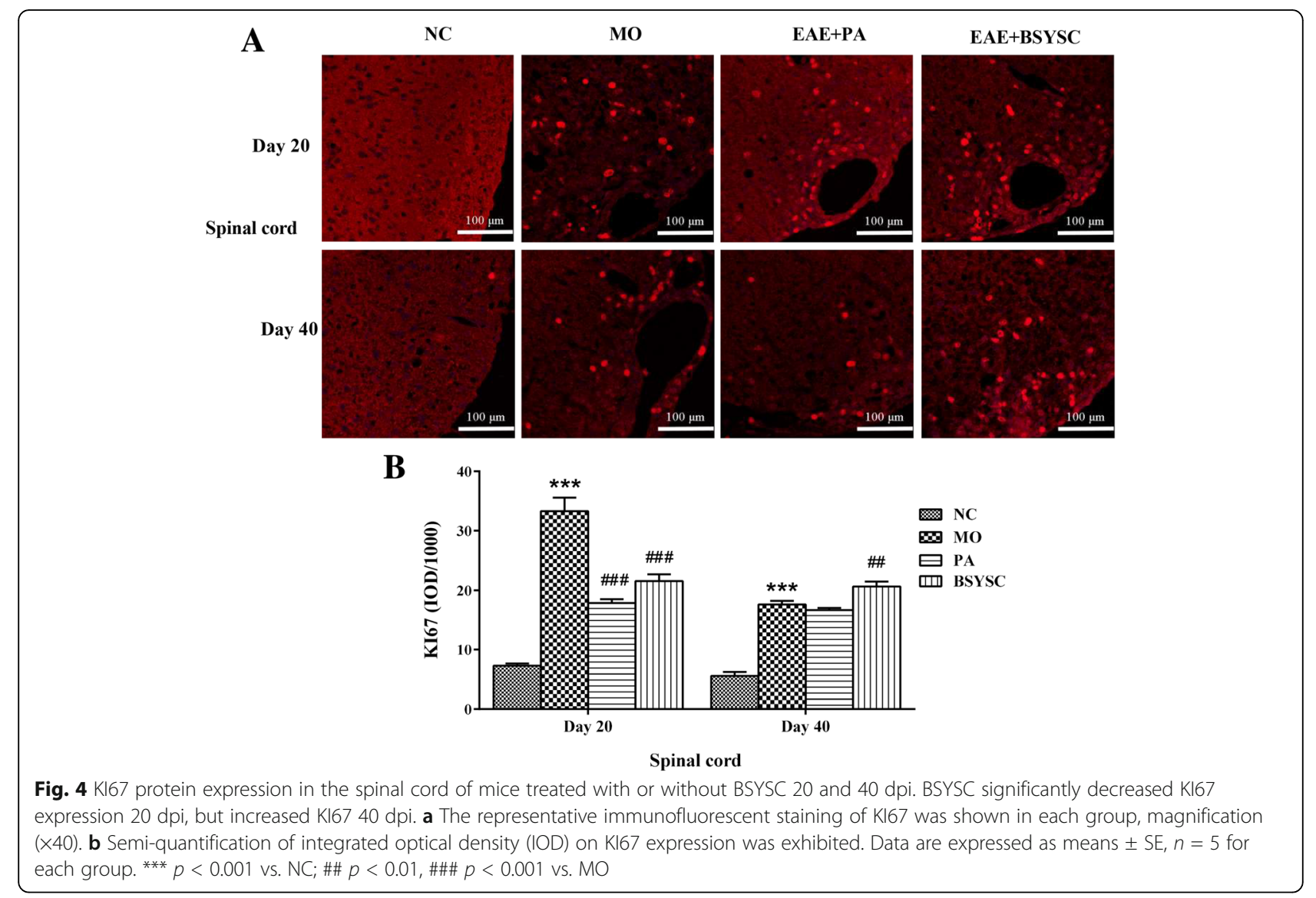

BSYSC mice as compared with the MO mice $(P<0.05$ or $P<0.01)$, RhoA was also decreased in brain $40 \mathrm{dpi}$ and spinal cord 20 dpi in PA mice $(P<0.01)$. It was worth mentioning that the effect of BSYSC was better than that of PA $(P<0.05$, Fig. 7c). ROCK protein was increased markedly in brain $20 \mathrm{dpi}$ and spinal cord 40 dpi in MO mice as compared with the NC mice $(P<0.05)$. A significant reduction of ROCK protein was observed in brain $40 \mathrm{dpi}$ in both PA and BSYSC mice as compared with the MO mice $(P<0.01)$, and ROCK was also decreased in spinal cord 20 and 40 dpi in BSYSC mice $(P<0.05$, Fig. $7 \mathrm{~d})$.

\section{Discussion}

In our previous studies, the characteristics of EAE mice were confirmed by the increase in neurological function scores, the existence of inflammatory infiltrates in brain and spinal cord, and damage to axons and the myelin sheath. It also appeared the immune imbalance in Th1/ Th2 and Th17/Treg cells. However, BSYSC treatment could improve the clinical symptoms and neurological dysfunction, reduce the inflammatory response and regulate the cellular immunity. These findings indicated that BSYSC had the neuroprotective effects in EAE mice
$[22,23,25]$. In the present study, we investigated the effects of BSYSC on reducing axonal injury and promoting axonal repair in EAE mice. We also explored further the regulation of $\mathrm{BSYSC}$ on $\mathrm{NogoA} / \mathrm{NgR}$ and its signaling pathways RhoA/ROCK.

PGP9.5 is a specific ubiquitin-hydroxy-enzyme in nerve fibers, which widely distributed in neuron, nerve fibers and many kinds of neuroendocrine cells [26, 27]. It is transported through slow axoplasmic transport in axons of nerve cell, and is the highest content of soluble protein in neurons $[28,29]$. Tau is a kind of neuron microtubule associated protein, which mainly concentrates in the axons [30-32]. It has the function of stabilizing the axonal microtubules and maintaining the axonal transport. The study found that PGP9.5 expression was reduced in rats after spinal cord injury [33]. The excessive Tau protein damaged the axoplasmic transport and caused the degeneration of neurons [34-36]. The abnormal phosphorylation of Tau protein was related with the absence of neurons and axons in chronic remission of recurrent EAE and secondary development of MS, leading to tissue degeneration, making disease progression to chronic stage [37]. In the current study, PGP9.5 protein expression 


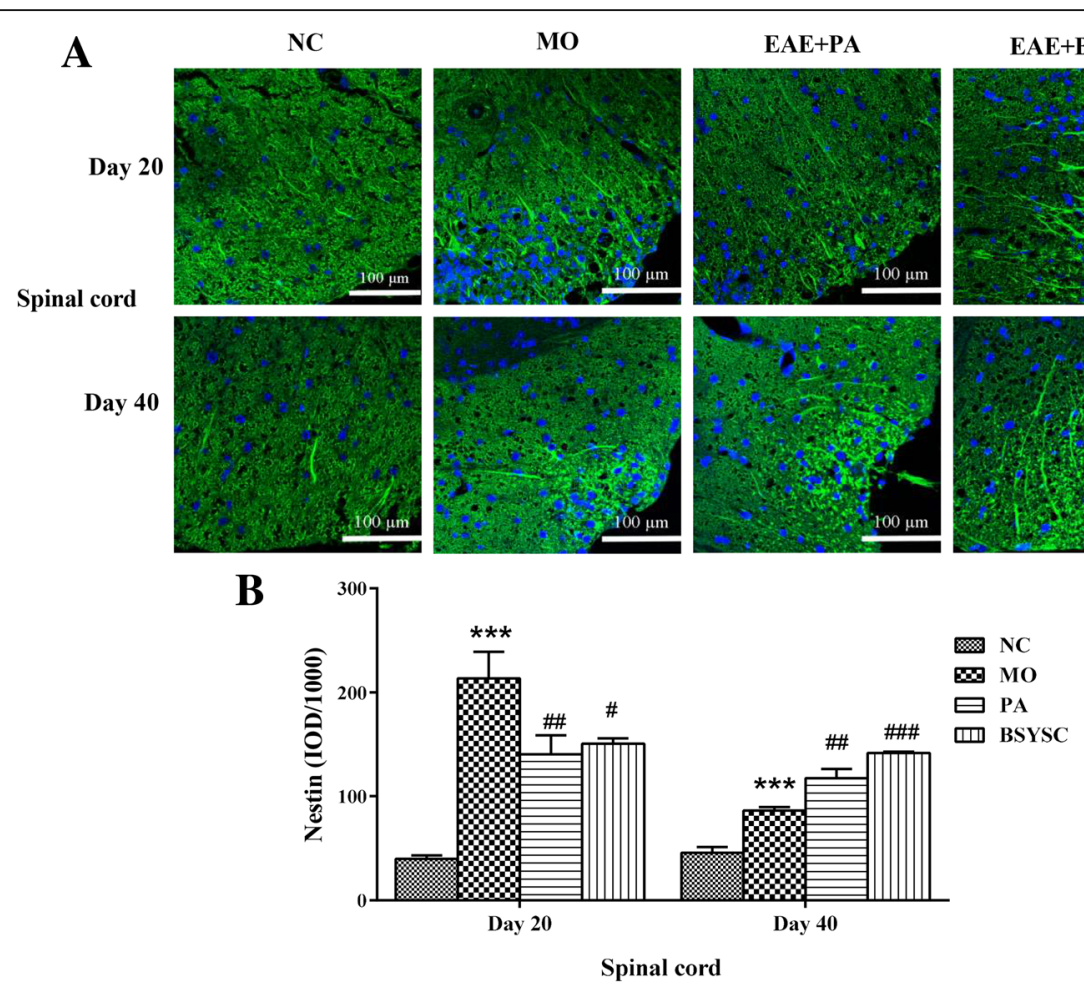

Fig. 5 Nestin expression in the spinal cord of mice treated with or without BSYSC 20 and 40 dpi. A significant decreased Nestin expression was found in the spinal cord of $20 \mathrm{dpi}$ in BSYSC mice, but increased Nestin was found $40 \mathrm{dpi}$. a The representative immunofluorescent staining of Nestin was shown in each group, magnification $(\times 40)$. b Semi-quantification of integrated optical density (IOD) on Nestin expression was exhibited. Data are expressed as means $\pm \mathrm{SE}, n=5$ for each group. ${ }^{* * *} p<0.01$ vs. NC; \# $p<0.05$, \#\# $p<0.01$, \#\#\# $p<0.001$ vs. MO

was significantly decreased, but p-Tau was markedly increased in brain or spinal cord of EAE mice during acute and/or remission phase, indicating the axon and myelin sheath were seriously injured in the whole pathological process. After treatment of BSYSC, the levels of PGP9.5 protein were increased obviously, and p-Tau were decreased markedly, which indicated that the formula had the effect of reducing the nerve injury. This effect may be related to the reduction of immune inflammation. Indeed, our previous studies found that BSYSC regulated the ratio of CD4 + IL-17+/CD4 + CD25 + FoxP3 $+\mathrm{T}$ cells in the spleen, decreased the cytokines such as IL17A, IL-6, IL-23 in the brain and mediated the balance of Th17/Treg cells [22, 23, 25].

GAP-43 is a kind of fast transport membrane protein, which is closely related to the neural development, axonal regeneration and synaptic reconstruction $[38,39]$. It is found that GAP-43 is highly expressed in the axonal regeneration and synaptic growth after brain injury [40-42]. Therefore, GAP43 is used as a marker of axonal regrowth and synaptogenesis. Our results showed BSYSC increased the expression of GAP-43 in the brain and spinal cord of EAE mice, and the increase in GAP-43 with BSYSC thereby indicates its effects of promoting axon growth.
As a nuclear proliferation antigen, KI67 serves as a cell proliferation marker. Nestin is a marker of neural stem cells, which highly expresses in the mammalian neural precursor and is able to differentiate into neurons and glia cells. Various potentials of neural stem cells demonstrated including migrating to the damage regions to fill and replace impaired neural cells. Our results showed that KI67 and Nestin in the spinal cord were significantly enhanced after BSYSC treatment 40 dpi in EAE mice, revealing the underlying potential of BSYSC on enhancing neural stem cell proliferation. Neural stem cells can be differentiated into different phenotypes, repairing injured neurons, glial cells and axons [43, 44], thus promoting functional recovery in EAE mice. Whereas no statistical difference of KI67 and Nestin was found in the brain in this experiment, this might be related to the severity of the inflammation and demyelination in spinal cord of EAE mice [45].

A large number of studies have found that NogoA is a membrane protein in the CNS restricting neurite growth and synaptic plasticity via the extracellular domain (Nogo-66), it exerts the inhibitory activity by binding to a neuronal receptor complex containing NgR, p75NTR and LINGO-1 [46-48], and activates the downstream signaling pathways of RhoA/ROCK, acts on the actin 


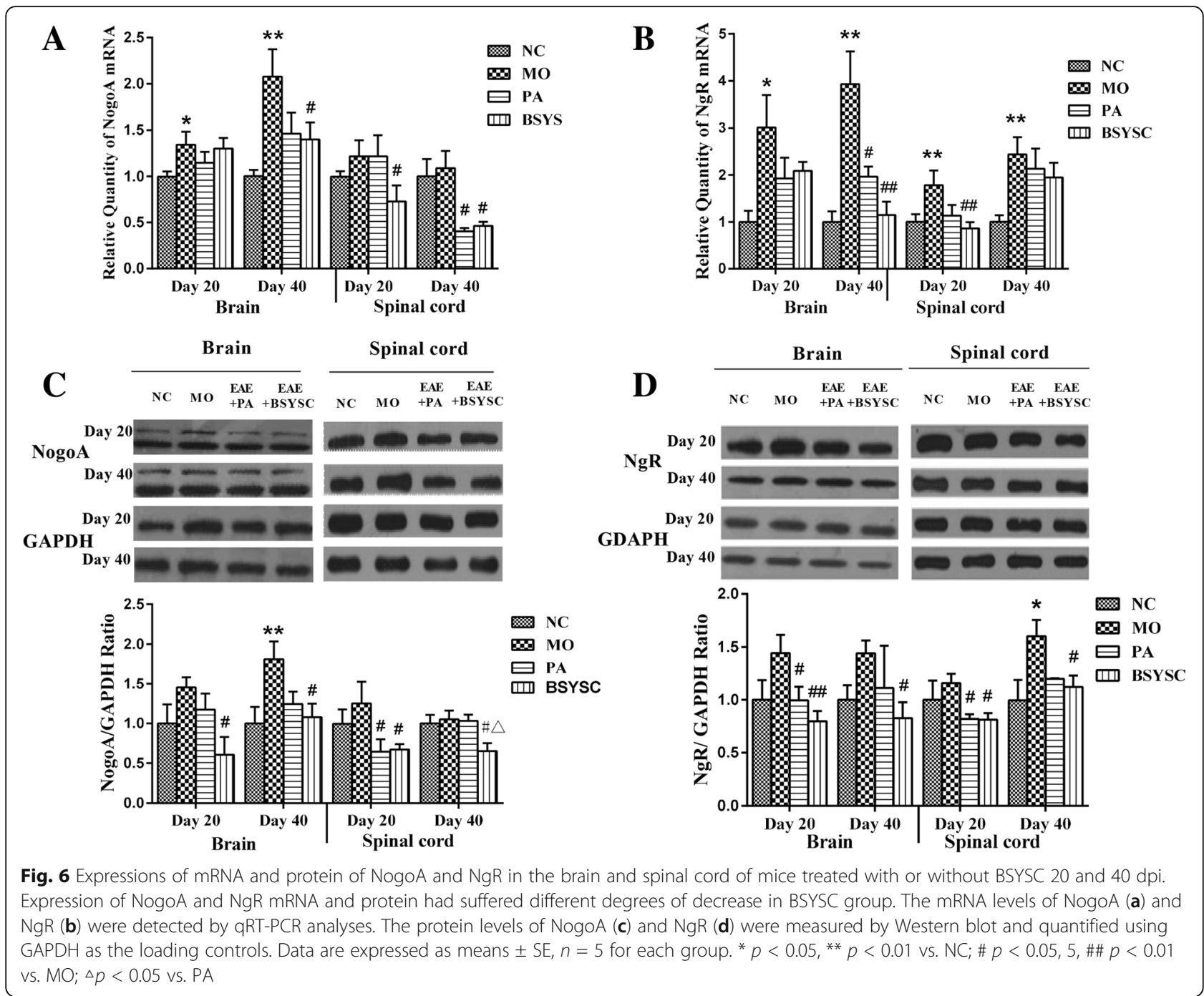

cytoskeleton system to cause growth cone collapse and neurite outgrowth inhibition [49-51]. The studies have also demonstrated that NogoA/NgR and its RhoA/ ROCK signaling pathways are involved in the development of MS or EAE. NogoA expression was markedly upregulated in surviving oligodendrocytes at the edge of chronic active demyelinating lesions of MS, whereas $\mathrm{NgR}$ was found to be significantly increased in reactive astrocytes and microglia/macrophages in these lesions [52]. TROY and LINGO-1 was also greatly enhanced in astrocytes and microglia in MS lesions [52, 53]. NogoA and RhoA are strongly expressed in brain tissues of MS and EAE rats $[54,55]$. In this study, NogoA, NgR, RhoA and ROCK mRNA and protein expressions in the brain and/or spinal cord of mice were significantly increased at acute and/or remission stage. Our data were consistent with these above described findings [56]. It indicates that a large number of inhibitory factors were produced in the lesions of axon and myelin which activate RhoA/
ROCK signaling pathways. Up to 40 days, the expressions of inhibitory factors were still increased, speculating that the degeneration of nerve existed in the whole progress of the disease. Therefore, blocking RhoA/ ROCK signaling can reverse the inhibitory effects of these molecules on axon outgrowth and functional recovery. Some scholars use ROCK inhibitors such as Y39983 and WAR-5 to treat EAE animal. Y-39983 attenuates EAE condition via inhibition of demyelination [57]. WAR-5 selectively suppresses the expression of ROCK II in spleen, brain and spinal cord of EAE mice, especially in spinal cord, accompanied by decreased expression of Nogo [58]. Here, we found that BSYSC can not only significantly reduce the nerve regeneration inhibitory factors NogoA and NgR but also inhibit its signaling pathways RhoA and ROCK. We also found that the changes of the above indexes were slight different in brain and spinal cords during the disease course, the explanation for this could be the formula treatment 


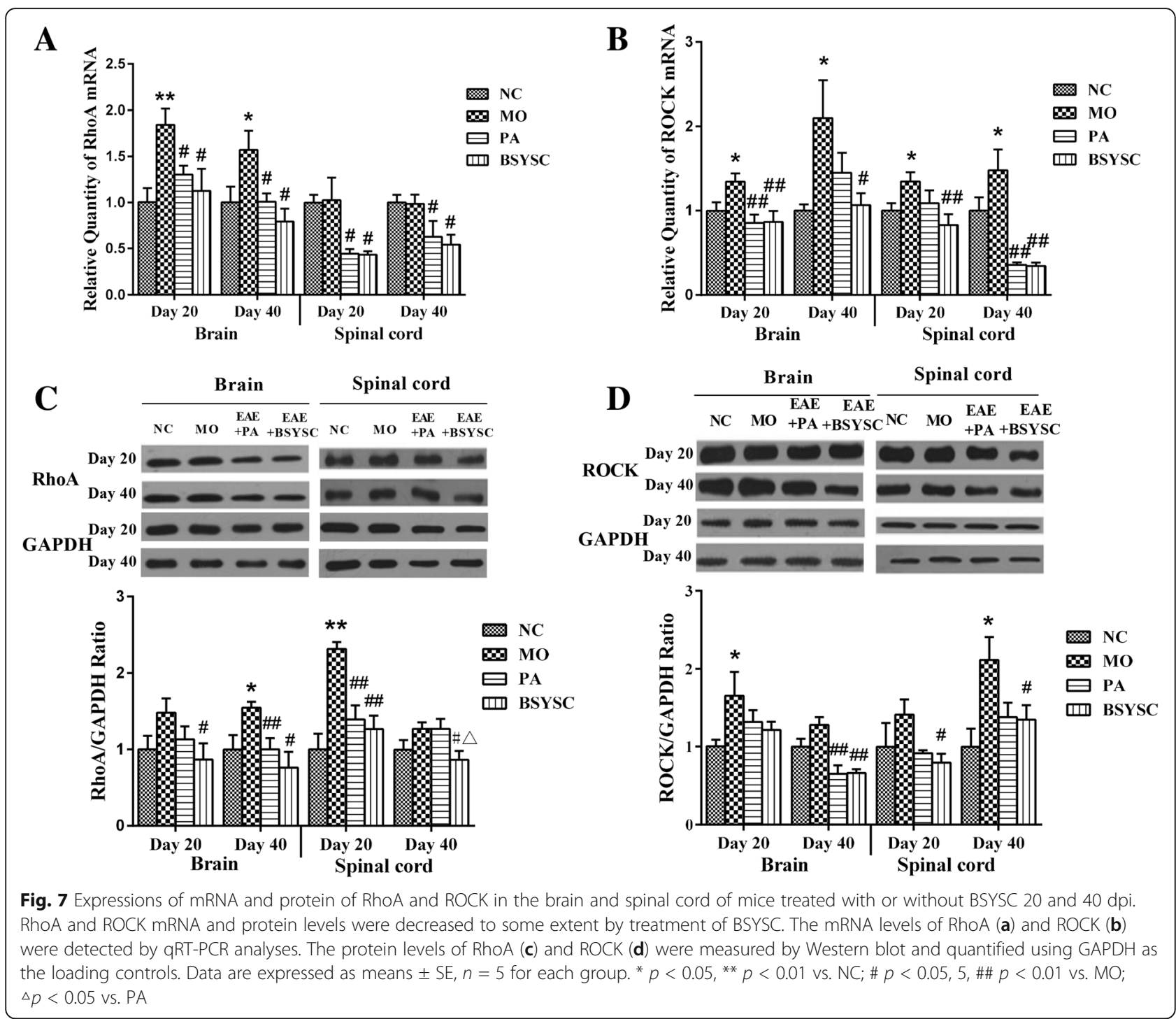

functioned more effectively at the early stage and tended to maximize the effects at $20 \mathrm{dpi}$ or later, or simply due to the sensitivity of our detection methodology was not sufficient to resolve the potential differences quantitatively at this point.

Recent studies have found that many Chinese medical formulas have the effects of moderating NogoA/NgR [59]. Several herbs in BSYSC that we studied were also known to play important roles in inhibitory signal pathway. For examples, gastrodin, the main constituent of Gastrodiae Rhizoma, down-regulated the expression of the neuronal cytoskeleton remodeling-related negative regulators Slit1 and RhoA in the hippocampus of rat model of depression [60], San-Huang-Xie-Xin-Tang containing Rhei Radix et Rhizoma attenuated ROCK-II protein expression in U46619-induced primary pulmonary smooth muscle cells [61]; additionally, Leonuri Herba, Scorpio and Gastrodiae Rhizoma were shown have neuroprotective and neuroregenerative effect in vivo and in vitro [62-65]. Moreover, the molecules identified in BSYSC like forsythiaside, acteoside were reported for neuroprotective effects [66-71]. These findings strongly implied that the effects of BSYSC in MS or EAE animal model, could be cooperative action with multiple compounds, also involved cross components worked together for the common effects. The exact roles of the active components for treatment of EAE need to be further investigated.

\section{Conclusions}

In summary, BSYSC exhibited the therapeutic effect on inducing neurogenesis and axon growth after axonal injury in EAE mice, which might be attributable to the modulation of NogoA/NgR and RhoA/ROCK. These findings provided new insights into the mechanism of BSYSC in the treatment of MS. 


\section{Abbreviations}

BSYSC: Bu Shen Yi Sui Capsule; CFA: Complete Freund' adjuvant; CNS: Central nervous system; EAE: Experimental autoimmune encephalomyelitis; GTPase: Guanosine triphosphatase; IF: Immunofluorescence; MAG: Myelin-associated glycoprotein; MOG: Myelin oligodendrocyte glycoprotein; MS: Multiple sclerosis; NgR: Nogo-66 receptor; OMgp: Oligodendrocyte myelin glycoprotein; PA: Prednisone acetate; PTX: Pertussis toxin; QRT-PCR: Real-time quantitative reverse transcription-polymerase chain reaction; SE: standard error; TCM: Traditional Chinese medicine

\section{Acknowledgments}

Not applicable.

\section{Funding}

This study was financially supported by the National Natural Science Foundation of China (No. 81573898, No. 81473640 and No. 81273742), the Scientific Research Key Program of Beijing Municipal Commission of Education (KZ201310025023) and the Program of Changcheng Scholars for the Importation and Development of High-Caliber Talents Project of Beijing Municipal Institutions (CIT\&TCD20140329).

\section{Availability of data and materials}

All data supporting the conclusions of this article are included within the article.

\section{Authors' contributions}

LF, YW, QZ, and TY participated in the experiments. LF, YW and PZ performed data statistics and manuscript preparation. LW and YF supported the funding, designed the study and revised the manuscript. $\mathrm{HZ}$ and $\mathrm{QZ}$ provided theoretical and technical guidance. $Y Z, F Q, K L, Z C, J L$ and $N Z$ participated in experimental preparation and statistical analysis. All authors read and approved the final manuscript.

\section{Ethics approval}

All experimental animal procedures were performed in strict accordance with Ethics Committee of Capital Medical University.

\section{Consent for publication}

Not applicable.

\section{Competing interests}

The authors declare that they have no competing interests.

\section{Publisher's Note}

Springer Nature remains neutral with regard to jurisdictional claims in published maps and institutional affiliations.

\section{Author details \\ ${ }^{1}$ School of Traditional Chinese Medicine, Beijing Key Lab of TCM Collateral Disease Theory Research, Capital Medical University, Beijing 100069, People's Republic of China. 'Beijing Tian Tan Hospital, Capital Medical University, Beijing 100050, People's Republic of China. ${ }^{3}$ Daxing Hospital Affiliated to Capital Medical University, Beijing 102600, People's Republic of China. ${ }^{4}$ Guang An Men Hospital of China Academy of Chinese Medical Sciences, Beijing 100053, People's Republic of China. ${ }^{5}$ Core Facility Center, Capital Medical University, Beijing 100069, People's Republic of China.}

\section{Received: 14 March 2017 Accepted: 20 June 2017}

\section{Published online: 01 July 2017}

\section{References}

1. Herz J, Zipp F, Siffrin V. Neurodegeneration in autoimmune CNS inflammation. Exp Neurol. 2010:225:9-17.

2. Gold R, Linington $\mathrm{C}$, Lassmann $\mathrm{H}$. Understanding pathogenesis and therapy of multiple sclerosis via animal models: 70 years of merits and culprits in experimental autoimmune encephalomyelitis research. Brain. 2006:129:1953-71

3. Wu F, Cao W, Yang Y, Liu A. Extensive infiltration of neutrophils in the acute phase of experimental autoimmune encephalomyelitis in C57BL/6 mice. Histochem Cell Biol. 2010;133:313-22.
4. Huang SY, Tobyne SM, Nummenmaa A, Witzel T, Wald LL, McNab JA, et al. Characterization of axonal disease in patients with multiple sclerosis using high-gradient-diffusion MR imaging. Radiology. 2016;151582

5. Simmons SB, Pierson ER, Lee SY, Goverman JM. Modeling the heterogeneity of multiple sclerosis in animals. Trends Immunol. 2013;34:410-22.

6. Baker D, Gerritsen W, Rundle J, Amor S. Critical appraisal of animal models of multiple sclerosis. Mult Scler. 2011;17:647-57.

7. Vogt J, Paul F, Aktas O, Muller-Wielsch K, Dorr J, Dorr S, et al. Lower motor neuron loss in multiple sclerosis and experimental autoimmune encephalomyelitis. Ann Neurol. 2009;66:310-22.

8. Seehusen F, Baumgartner W. Axonal pathology and loss precede demyelination and accompany chronic lesions in a spontaneously occurring animal model of multiple sclerosis. Brain Pathol. 2010;20:551-9.

9. Kerschensteiner M, Bareyre FM, Buddeberg BS, Merkler D, Stadelmann C. Bruck W, et al. Remodeling of axonal connections contributes to recovery in an animal model of multiple sclerosis. J Exp Med. 2004; 200:1027-38

10. Sandvig A, Berry M, Barrett LB, Butt A, Logan A. Myelin-, reactive glia-, and scar-derived CNS axon growth inhibitors: expression, receptor signaling, and correlation with axon regeneration. Glia. 2004;46:225-51.

11. Wang KC, Kim JA, Sivasankaran R, Segal R, He ZG. p75 interacts with the Nogo receptor as a co-receptor for Nogo. MAG and OMgp Nature. 2002:420:74-8.

12. Tang BL. Inhibitors of neuronal regeneration: mediators and signaling mechanisms. Neurochem Int. 2003:42:189-203.

13. Domeniconi M, Filbin MT. Overcoming inhibitors in myelin to promote axonal regeneration. J Neurol Sci. 2005;233:43-7.

14. Cao Z, Gao Y, Deng K, Williams G, Doherty P, Walsh FS. Receptors for myelin inhibitors: structures and therapeutic opportunities. Mol Cell Neurosci. 2010;43:1-14.

15. Fujita $Y$, Yamashita T. Axon growth inhibition by RhoA/ROCK in the central nervous system. Front Neurosci. 2014;8:338.

16. Ontaneda D, Hyland M, Cohen JA. Multiple sclerosis: new insights in pathogenesis and novel therapeutics. Annu Rev Med. 2012;63:389-404.

17. Liu J, Gao Y. Kan B-h. Zhou L Systematic review and meta-analysis of randomized controlled trials of Chinese herbal medicine in treatment of multiple sclerosis Journal of Chinese Integrative Medicine. 2012;10:141-53.

18. Kochs L, Wegener S, Suhnel A, Voigt K, Zettl UK. The use of complementary and alternative medicine in patients with multiple sclerosis: a longitudinal study. Complement Ther Med. 2014:22:166-72.

19. Ling F, Yong-ping F, Hui Z, Lei W. Review on prevention and treatment of multiple sclerosis by Bu Shen Yi sui formula. Guid J Tradit Chin Med Pharm. 2013;19:108-10.

20. Zhou L, Fan Y. Randomized trial of erhuangfang for relapsing multiple sclerosis. Neurol Res. 2015:37:633-7.

21. Li K, Fan Y, Yang T, Wang L. Mechanism of Erhuang capsule for treatment of multiple sclerosis. Neural Regen Res. 2013;8:523-31.

22. Fang L, Zheng Q, Yang T, Zhao H, Zhang Q, Li K, et al. Bushen Yisui capsule ameliorates axonal injury in experimental autoimmune encephalomyelitis. Neural Regen Res. 2013:8:3306-15.

23. Zheng $Q$, Yang $T$, Fang $L$, Liu $L$, Liu $H$, Zhao $H$, et al. Effects of $B u$ Shen Yi sui capsule on Th17/Treg cytokines in C57BL/6 mice with experimental autoimmune encephalomyelitis. BMC Complement Altern Med. 2015;15:60.

24. Pharmacopoeia NCoC: Pharmacopoeia of the People's Republic of China 2015(1st set). Beijing: China Medical Science Press; 2015.

25. Liu $Y$, Zhao $H$, Zhang J, Zhang $P$, Li M, Qi F, et al. The regulatory effect of Liuwei Dihuang pills on cytokines in mice with experimental autoimmune encephalomyelitis. Am J Chinese Med. 2012;40:295-308.

26. Hasan W, Cowen T, Barnett PS, Elliot E, Coskeran P, Bouloux PM. The sweating apparatus in growth hormone deficiency, following treatment with r-hGH and in acromegaly. Auton Neurosci. 2001;89:100-9.

27. Loo LS, Ng YK, Zhu YZ, Lee HS, Wong PT. Cortical expression of endothelin receptor subtypes a and $\mathrm{B}$ following middle cerebral artery occlusion in rats. Neuroscience. 2002;112:993-1000

28. Sidebotham EL, Woodward MN, Kenny SE, Lloyd DA, Vaillant CR, Edgar DH. Assessment of protein gene product 9.5 as a marker of neural crest-derived precursor cells in the developing enteric nervous system. Pediatr Surg Int 2001;17:304-07.

29. Young HM, Hearn CJ, Newgreen DF. Embryology and development of the enteric nervous system. Gut. 2000;47 Suppl 4:iv12-4; discussion iv26. 
30. Cleveland DW, Hwo SY, Kirschner MW. Physical and chemical properties of purified tau factor and the role of tau in microtubule assembly. J Mol Biol. 1977;116:227-47.

31. Binder LI, Frankfurter A, Rebhun LI. The distribution of tau in the mammalian central nervous system. J Cell Biol. 1985;101:1371-8.

32. Panda D, Goode BL, Feinstein SC, Wilson L. Kinetic stabilization of microtubule dynamics at steady state by tau and microtubule-binding domains of tau. Biochemistry. 1995;34:11117-27.

33. Zhou C, Wang Y, Hou B, Liu M, Shi Q. Effects of Yiqi Huayu Fang on rat's lumber nerve root during regeneration and repair of nerve. Zhongguo Lin Chuang Kang Fu. 2004;8:192-4

34. Mandelkow E-M, Biernat J, Drewes G, Gustke N, Trinczek B, Mandelkow E. Tau domains, phosphorylation, and interactions with microtubules. Neurobiol Aging. 1995;16:355-62.

35. Saragoni L, Hernandez P, Maccioni RB. Differential association of tau with subsets of microtubules containing posttranslationally-modified tubulin variants in neuroblastoma cells. Neurochem Res. 2000;25:59-70.

36. Yang Y, Yang XF, Wang YP, Tian Q, Wang XC, Li HL, et al. Inhibition of protein phosphatases induces transport deficits and axonopathy. J Neurochem. 2007;102:878-86.

37. Anderson J, Hampton D, Patani R, Pryce G, Crowther R, Reynolds R, et al. Abnormally phosphorylated tau is associated with neuronal and axonal loss in experimental autoimmune encephalomyelitis and multiple sclerosis. Brain. 2008;131:1736-48.

38. Carmichael ST. Plasticity of cortical projections after stroke. Neuroscientist. 2003;9:64-75

39. Grasselli G, Strata P. Structural plasticity of climbing fibers and the growth-associated protein GAP-43. Front Neural Circuits. 2013;7:25.

40. Tolner EA, van Vliet EA, Holtmaat AJ, Aronica E, Witter MP, da Silva FH, et al. GAP-43 mRNA and protein expression in the hippocampal and parahippocampal region during the course of epileptogenesis in rats. Eur J Neurosci. 2003;17:2369-80.

41. Iwata M, Shirayama Y, Ishida H, Kawahara R. Hippocampal synapsin I, growth-associated protein-43, and microtubule-associated protein-2 immunoreactivity in learned helplessness rats and antidepressant-treated rats. Neuroscience. 2006;141:1301-13.

42. Jutapakdeegul N, Afadlal S, Polaboon N, Phansuwan-Pujito P, Govitrapong P. Repeated restraint stress and corticosterone injections during late pregnancy alter GAP-43 expression in the hippocampus and prefrontal cortex of rat pups. Int J Dev Neurosci. 2010;28:83-90.

43. Hirata K, Kanemaru T, Minohara M, Togo A, Kira J. Accumulation of stressrelated proteins within the glomeruli of the rat olfactory bulb following damage to olfactory receptor neurons. Arch Histol Cytol. 2008;71:265-77.

44. Hoveizi E, Tavakol S, Ebrahimi-Barough S. Neuroprotective effect of transplanted neural precursors embedded on PLA/CS scaffold in an animal model of multiple sclerosis. Mol Neurobiol. 2015;51:1334-42.

45. Gao Z, Nissen JC, Legakis L, Tsirka SE. Nicotine modulates neurogenesis in the central canal during experimental autoimmune encephalomyelitis. Neuroscience. 2015;297:11-21.

46. Chen MS, Huber AB, van der Haar ME, Frank M, Schnell L, Spillmann AA, et al. Nogo-a is a myelin-associated neurite outgrowth inhibitor and an antigen for monoclonal antibody IN-1. Nature. 2000;403:434-9.

47. Mi S, Lee X, Shao Z, Thill G, Ji B, Relton J, et al. LINGO-1 is a component of the Nogo-66 receptor/p75 signaling complex. Nat Neurosci. 2004;7:221-8.

48. Niederost B, Oertle T, Fritsche J, McKinney RA, Bandtlow CE. Nogo-a and myelin-associated glycoprotein mediate neurite growth inhibition by antagonistic regulation of RhoA and Rac1. J Neurosci. 2002;22:10368-76.

49. Josephson A, Trifunovski A, Widmer HR, Widenfalk J, Olson L, Spenger C. Nogo-receptor gene activity: cellular localization and developmental regulation of mRNA in mice and humans. J Comp Neurol. 2002;453:292-304.

50. Bustelo XR, Sauzeau V, Berenjeno IM. GTP-binding proteins of the rho/Rac family: regulation, effectors and functions in vivo. BioEssays. 2007;29:356-70.

51. Kubo T, Hata K, Yamaguchi A, Yamashita T. Rho-ROCK inhibitors as emerging strategies to promote nerve regeneration. Curr Pharm Design. 2007;13:2493-9.

52. Satoh J-I, Onoue H, Arima K, Yamamura T. Nogo-a and nogo receptor expression in demyelinating lesions of multiple sclerosis. J Neuropath Exp Neur. 2005;64:129-38.

53. Satoh J, Tabunoki H, Yamamura T, Arima K, Konno H. TROY and LINGO-1 expression in astrocytes and macrophages/microglia in multiple sclerosis lesions. Neuropathol Appl Neurobiol. 2007;33:99-107.
54. Karnezis T, Mandemakers W, McQualter JL, Zheng BH, Ho PP, Jordan KA, et al. The neurite outgrowth inhibitor NogoA is involved in autoimmunemediated demyelination. Nat Neurosci. 2004;7:736-44.

55. Zhang Z, Schittenhelm J, Meyermann R, Schluesener HJ. Lesional accumulation of RhoA(+) cells in brains of experimental autoimmune encephalomyelitis and multiple sclerosis. Neuropathol Appl Neurobiol. 2008; 34:231-40.

56. Theotokis $P$, Lourbopoulos A, Touloumi O, Lagoudaki R, Kofidou E, Nousiopoulou E, et al. Time course and spatial profile of Nogo-a expression in experimental autoimmune encephalomyelitis in C57BL/6 mice. J Neuropathol Exp Neurol. 2012;71:907-20.

57. Gao C, Huang L, Long Y, Zheng J, Yang J, Pu S, et al. Y-39983, a selective rho-kinase inhibitor, attenuates experimental autoimmune encephalomyelitis via inhibition of demyelination. Neuroimmunomodulation. 2013:20:334-40.

58. Li YH, Yu JZ, Xin YL, Feng L, Chai Z, Liu JC, et al. Protective effect of a novel rho kinase inhibitor WAR-5 in experimental autoimmune encephalomyelitis by modulating inflammatory response and neurotrophic factors. Exp Mol Pathol. 2015;99:220-8.

59. Qin XD, Kang LY, Liu Y, Huang Y, Wang S, Zhu JQ. Chinese Medicine's intervention effect on Nogo-a/NgR. Evid Based Complement Alternat Med. 2012:2012:528482

60. Chen WC, Lai YS, Lin SH, Lu KH, Lin YE, Panyod S, et al. Anti-depressant effects of Gastrodia elata Blume and its compounds gastrodin and 4hydroxybenzyl alcohol, via the monoaminergic system and neuronal cytoskeletal remodeling. J Ethnopharmacol. 2016;182:190-9.

61. Tsai HH, Chen IJ, Lo YC. Effects of san-Huang-Xie-Xin-Tang on U46619induced increase in pulmonary arterial blood pressure. J Ethnopharmacol. 2008;117:457-62.

62. Shi XR, Hong ZY, Liu HR, Zhang YC, Zhu YZ. Neuroprotective effects of SCM198 on 6-hydroxydopamine-induced behavioral deficit in rats and cytotoxicity in neuronal SH-SY5Y cells. Neurochem Int. 2011;58:851-60.

63. Wang T, Wang SW, Zhang Y, Wu XF, Peng Y, Cao Z, et al. Scorpion venom heat-resistant peptide (SVHRP) enhances neurogenesis and neurite outgrowth of immature neurons in adult mice by up-regulating brainderived neurotrophic factor (BDNF). PLoS One. 2014;9:e109977.

64. Baral S, Pariyar R, Yoon CS, Kim DC, Yun JM, Jang SO, et al. Effects of Gastrodiae rhizoma on proliferation and differentiation of human embryonic neural stem cells. Asian Pac J Trop Med. 2015;8:792-7.

65. Zhang R, Peng Z, Wang H, Xue F, Chen Y, Wang Y, et al. Gastrodin ameliorates depressive-like behaviors and up-regulates the expression of BDNF in the hippocampus and hippocampal-derived astrocyte of rats. Neurochem Res. 2014;39:172-9.

66. Huang C, Lin Y, Su H, Ye D. Forsythiaside protects against hydrogen peroxide-induced oxidative stress and apoptosis in PC12 cell. Neurochem Res. 2015;40:27-35.

67. Wang HM, Wang LW, Liu XM, Li CL, Xu SP, Farooq AD. Neuroprotective effects of forsythiaside on learning and memory deficits in senescenceaccelerated mouse prone (SAMP8) mice. Pharmacol Biochem Behav. 2013; 105:134-41.

68. Kim JM, Kim S, Kim DH, Lee CH, Park SJ, Jung JW, et al. Neuroprotective effect of forsythiaside against transient cerebral global ischemia in gerbil. Eur J Pharmacol. 2011;660:326-33.

69. Xiong L, Mao S, Lu B, Yang J, Zhou F, Hu Y, et al. Osmanthus fragrans Flower extract and Acteoside protect against d-galactose-induced aging in an ICR mouse model. J Med Food. 2016;19:54-61.

70. Peng XM, Gao L, Huo SX, Liu XM, Yan M. The mechanism of memory enhancement of Acteoside (Verbascoside) in the senescent mouse model induced by a combination of D-gal and AICl3. Phytother Res. 2015;29:1137-44.

71. Esposito E, Dal Toso R, Pressi G, Bramanti P, Meli R, Cuzzocrea S. Protective effect of verbascoside in activated C6 glioma cells: possible molecular mechanisms. Naunyn Schmiedeberg's Arch Pharmacol. 2010;381:93-105. 